\title{
Von der reaktiven zur proaktiven Therapie
}

- Bei der Behandlung der atopischen Dermatitis vollzieht sich ein Paradigmenwechsel: weg von der reaktiven Therapie, die erst beim Auftreten eines Schubs einsetzt, hin zur proaktiven Therapie, die auch in den schubfreien Intervallen fortgeführt wird. Der Grund für diese Umstellung ist die Erkenntnis, dass bei Neurodermitikern die Haut selbst in der schubfreien Zeit immer noch eine subklinische Entzündung in sich trägt. Daher besteht Bedarf für eine Langzeittherapie mit dem Ziel, die subklinische Entzündung unter Kontrolle zu bringen, Ekzemschübe zu verhindern und die erkran- kungsfreien Intervalle zu verlängern, erklärte Dr. Diamant Thaci, Frankfurt/Main. Mit einer solchen proaktiven Therapie soll die Haut von ihrem instabilen in einen „normalen" Zustand überführt werden.

Die beiden CONTROL-Studien bestätigen den Nutzen der Erhaltungstherapie mit Tacrolimus (Protopic ${ }^{\circledR}$ ): 257 Erwachsene mit mittelschwerer oder schwerer atopischer Dermatitis trugen den topischen Calcineurininhibitor ein Jahr lang auch zwischen den Schüben zweimal pro Woche auf die zuvor befallenen Hautareale auf. Ebenso konsequent wendeten sie eine Basistherapie mit

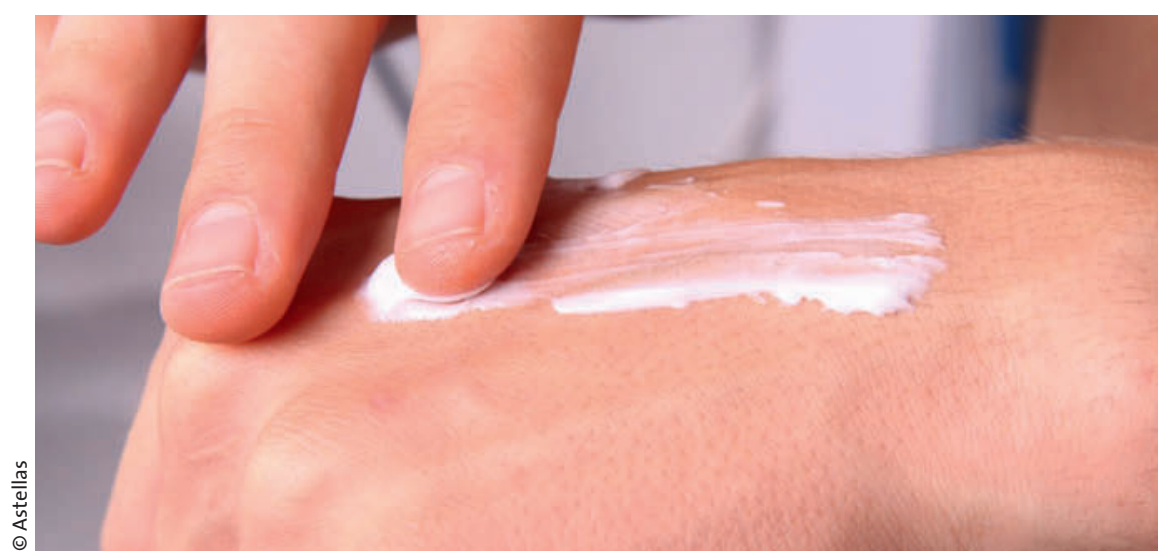

\section{Effektive CHE-Behandlung senkt Gesamttherapiekosten}

\begin{abstract}
— Unbehandelt beziehungsweise schlecht versorgt verursacht das chronische Handekzem (CHE) hohe Krankheitskosten: Direkte und indirekte Kosten summieren sich auf fast 10.000 Euro pro Patient und Jahr, dazu kommen ,intangible“ Kosten, die aus signifikanten Einbußen an Lebensqualität und hohem Leidensdruck der Patienten resultieren und nicht berechnet werden können. Das berichtete Prof. Dr. Matthias Augustin aus Hamburg.

Mit dem Vitamin-A-Derivat Alitretinoin (Toctino $^{\circledR}$ ) steht seit November 2008 eine Therapie zur Verfügung, die nicht nur therapeutisch, sondern auch wirtschaftlich sinnvoll ist: Aufgrund überzeugender Studiendaten zur Effektivität empfiehlt die Deutsche Der-
\end{abstract}

Sinnvolle Ergänzung bei atopischer Dermatitis: Erhaltungstherapie zwischen den Schüben.

einem lipidhaltigen Emollient an. Auf diese Weise wurde die Zahl der Ekzemschübe im Vergleich zur reaktiven Therapie signifikant reduziert. Etwa die Hälfte der Patienten erlitt während des Beobachtungszeitraums von einem Jahr keinen einzigen Schub. Bis zum ersten Schub verging etwa zehnmal soviel Zeit wie unter der reaktiven Therapie (142 vs. 15 Tage). Ähnliche Ergebnisse wurden auch bei Kindern beobachtet.

Größtes Problem scheint die Compliance der Patienten zu sein, die gerne „vergessen“, die Behandlung fortzusetzen, wenn sie keine Symptome haben. In diesen Fällen kann zum Beispiel ein intraindividueller Vergleich lehrreich sein, bei dem nur ein Arm proaktiv behandelt wird, empfahl Thaci. Wie lange die Therapie fortgeführt werden soll, sei immer individuell zu entscheiden. Ein Auslassversuch kann ausprobiert werden, wenn ein halbes Jahr lang kein Schub aufgetreten ist.

\section{jn}

Mittagsseminar „Proaktive Therapie mit Tacrolimus Salbe - Fortschritt in der Behandlung der atopischen Dermatitis, München, 28.7.2010 (Veranstalter: Astellas Pharma)

Autonomie mit weniger Zeitaufwand etwa für topische Therapien, Arztbesuche oder Klinikaufenthalte. Im Anschluss bewertet der Patient den Nutzen der Behandlung.,,84\% der Patienten erreichten den selbstdefinierten Nutzen durch Alitretinoin“, sagte Augustin. Dass durch die Effektivität der Therapie erhebliche Kosten eingespart werden können, zeigen Bewertungen der Wirtschaftlichkeit, die das Scottish Medicines Consortium (SMC) und das National Institute for Health and Clinical Excellence (NICE) 2009 durchgeführt haben: Sie errechneten Einsparpotenziale von 3,3 bis 3,7 Millionen Pfund für Großbritannien über fünf Jahre im Vergleich zu anderen CHE-Therapien und empfehlen die Therapie als hoch wirtschaftlich. $\quad$ koc

(Symposium „Rund um das Handekzem: Dermatologie zum Anfassen“, Hamburg, 2. Oktober 2010 (Veranstalter: Basilea Pharmaceutica Deutschland) 